\title{
A new methodological approach to nonverbal behavior analysis in cultural perspective
}

\author{
ALESSIA AGLIATI, ANTONIETTA VESCOVO, and LUIGI ANOLLI \\ University of Milan-Bicocca, Milan, Italy
}

\begin{abstract}
The measurement of human behavior is a complex task, both for psychologists and human sciences researchers and with respect to technology, since advanced and sophisticated instruments may have to be implemented to manage the plurality of variables involved. In this article, an observational study is presented in which a quantitative procedure, the external variables method (Duncan \& Fiske, 1977), was integrated with a structural analysis (Magnusson, 1993, 2000) in order to detect the hidden organization of nonverbal behavior in Italian and Icelandic interactions. To this aim. Theme software was introduced and employed. The results showed that both the frequency and the typology of gestures deeply change as a function of culture. Moreover, a high number of patterns was detected in both Italian and Icelandic interactions: They appeared to be complex sequences in which a huge number of events were constantly happening and recurring. In this domain, Theme software provides a methodological progression from the quantitative to the structural approach.
\end{abstract}

Human behavior is to be considered a complex system, given its variable and multicomponent nature. This complexity is due primarily to the plurality of systems that, together, constitute behavior. Verbal, nonverbal, and vocal modalities, in particular, contribute to the visible and observable features of human behavior and may be defined as external variables (Duncan \& Fiske, 1977).

Human behavior changes as a function of individual variables and factors relating both to stable features, such as personality traits and dispositions, and to contextual factors, such as current mood (Higgins, 2000).

In this respect, context acts as a strong behavioral organizer, with particular impact on human behavior in interactions. In fact, people display a consistent tendency to adapt their behavior to their interlocutor and to the interactive situation (Giles, Coupland, \& Coupland, 1991; Giles \& Smith, 1979; Goodwin, 2000; Ochs, 1992).

Moreover, culture provides an overall template for behavior in general, since it has been established that not only language, but also gestures and nonverbal communication systems in general are learned and developed through the socialization process (Birdwhistell, 1970).

In the light of this complexity, the measuring of human behavior represents an intriguing "challenge" not only for psychologists and human science researchers, but also for technology, since increasingly more advanced and sophisticated instruments are required to manage the plurality of variables involved.

Special thanks to Stefania Netti and Chiara Gusmani for their support in data collection. Correspondence concerning this article should be addressed to A. Agliati, University of Milan-Bicocca, Piazza dell'Ateneo Nuovo, 1, 20126 Milan, Italy (e-mail: alessia.agliati@unimib.it).

\section{The Hidden Organization of Behavior}

Recently, in the psychological sciences, observational methodologies have been shown to be the most promising techniques for the analysis of human behavior, capable of dealing with its complexity and variability (Anguera, 2003; Magnusson, 2000b). Observational methods are scientific procedures that reveal the occurrence of perceptible behaviors, allowing them to be formally recorded and quantified.

Observational methods also allow analysis of the relationships between these behaviors, such as sequential characteristics, association, and covariation. Behavior complexity, indeed, is not synonymous with chaos. On the contrary, behaviors in interactions are nonrandom, patterned, and synchronized in both timing and form; they are structured and deeply organized (Bernieri \& Rosenthal, 1991; Magnusson, 2000a). As Eibl-Eibesfeldt (1970) has stated, "behavior consists of patterns in time. Investigations of behavior deal with sequences that, in contrast to bodily characteristics, are not always visible" (p. 1). These hidden patterns are fundamental for an adequate and effective understanding of behavior organization, since they are the "building blocks" of its design. Hidden patterns recur during the interaction flow, giving rise to a behavioral regularity that is the origin of interaction rhythm. It is a sort of "behavioral dance" (Grammer, Kruck, \& Magnusson, 1998) that constitutes the background of the interaction and characterizes it, since the time structure and rhythm of behavior remain quite stable throughout interaction flow.

In this context, the hidden organization of behaviors poses challenging and interesting research problems and has been explicitly investigated only by a few laboratories, whose activity has been linked to, or based on, the seminal work of an Icelandic scholar who created a specific 
software for the detection of hidden behavioral patterns (Magnusson, 1993, 2000a). These studies have provided a starting point for a structural analysis of behavior, aimed at detecting the hidden organization of behaviors (Anolli, Riva, Duncan, \& Magnusson, 2005).

The main objective of the research described in this article was, in fact, the detection of hidden behavioral structure. Considering the different variability factors that influence human behavior, a key assumption of this study was that culture shapes the hidden organization of behavior displayed in interactions, as has been found in research on conversation within the pragmatic perspective (Goodwin, 1981).

\section{Culture and Time Structure of Behavior in Conversation}

Conversation, indeed, may be defined as the best context in which to measure behavior; it is a universal communication system, characterized by a deep and intrinsic organization, conforming to given social and cultural standards (Goodwin, 1981). Culture provides individuals with standards and resources with which to guide their participation in social life in general and in everyday conversations in particular. The cultural influence is demonstrated by the variability to be found in verbal and nonverbal behavior. Various experimental studies have described cultural specificities for gestures, both in typology and in production frequency, during conversation (Goodwin, 2000; Graham \& Argyle, 1975; Morris, 1977).

Culture also seems to influence the time structure of interaction, as has been revealed by research in the field of chronemics, which examines how culture affects and shapes the temporal rhythm of human behavior in general and the use of time in communicative settings in particular (Hall \& Hall, 1999). For instance, a cross-cultural comparative analysis of conversation highlighted how people from different cultures manage time in very different ways during the conversational stream (Grader, 2003).

Within psychology and sociology, time use in conversation has been the object of conversational analysis studies taking a qualitative approach, aimed at examining the organization of talk in action (Goodwin, 2002; Zimmerman \& West, 1975). Recently, behavior in conversation has been studied using a structural approach, aimed at discovering the hidden temporal organization of interaction via pattern detection (Bloomfield, Jonsson, Polman, Houlahan, \& O’Donoghue, 2005; Merten \& Schwab, 2005).

\section{T-Pattern Detection Using Theme Software}

Obviously, before any pattern is analyzed, it must first be detected. Since behavioral patterns are hidden in interaction time flow, they can be detected only by using specific technical procedures for interaction analysis. The main purpose of this article is to present a new instrument that is capable of identifying hidden real-time patterns in intra- and interindividual streams of behavioral events (Anolli et al., 2005).

The Theme software (Magnusson, 1993, 2000a) is the device that was used in the present study to detect hidden time patterns of nonverbal behavior units in Icelandic and Italian conversations.

Theme (Magnusson, 1993, 2000a) is a tool designed to assist in studying the structure of human face-to-face interactions by searching for a particular type of repeated time patterns, called T-patterns (type-patterns).

The Theme package consists of Theme Coder, a videocoding program enabling effective coding of each behavior occurrence, frame by frame. It collects and memorizes the beginning and ending of every occurrence of a behavior unit, detected by a human coder, on the basis of a specific behavior grid (VVT, variable value table).

\section{T-Pattern Detection}

In this study, Theme Coder was used to code nonverbal behavior units in interactions. The beginning and ending of each behavior was recorded and encoded. Each beginning and ending thus occurs at a discrete time point and gives rise to an event type. For instance, John begins smiling (in Theme's notation: john,b,smile) and Mary ends jumping (in Theme's notation: mary,e,jump) are event types referring to the behavior units smile and jump, respectively.

To assess the reliability of the scoring, $25 \%$ of the protocols were also coded by a second observer. Reliability was calculated as an index of concordance, using the McGrew (1972) formula; a concordance of $80 \%$ was observed between the coders.

The occurrences of all event types within the observation period under consideration constitute the so-called $\mathrm{T}$ (temporal) data (or T-data set), which is the input to the $\mathrm{T}$ (temporal) pattern detection algorithm. The T-pattern algorithm has been implemented in a specialized software package, called Theme (Magnusson, 2000a, 2005; for further information, see www.patternvision.com and www.noldus.com), which was used in the present study. A T-pattern is essentially a combination of event types occurring in the same order and with the time distances between consecutive pattern components remaining relatively invariant (i.e., the time difference between A and B will be $x \pm y$ ).

If one assumes that the letters in Figure 1 correspond to specific event types that appear on the line in proportion to the time of their occurrence, the line may be a visual rep-

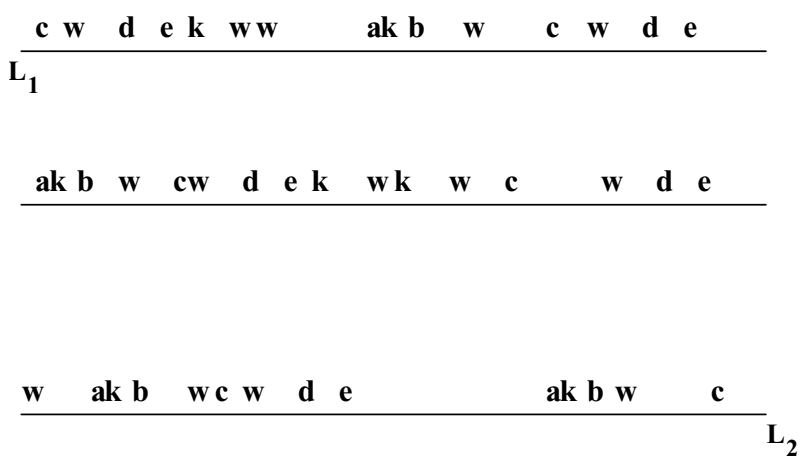

Figure 1. Stream of observed behavioral units. 
resentation of the temporal sequence of movements shown by a given subject during an interaction. In Figure 1, a sequence of four event types-A, B, C, and D-recurs but is masked by the occurrence of two other event types, $\mathrm{W}$ and $\mathrm{K}$. If an observer, even a highly expert one, were to visually inspect such data strings, it is unlikely that any kind of pattern would be detected. However, a T-pattern analysis would identify the repeated pattern A, B, C, and D, because of its consistent temporal structure. The T-pattern detection algorithm enables an analyst to discover repeated temporal patterns, even when various other event types occur between the components of the pattern, masking it from detection by the naked eye.

A schematic representation of a T-pattern is shown in Figure 2, which illustrates how a larger pattern $((\mathrm{AB})(\mathrm{CD}))$ is detected as a combination of two simpler patterns, $(\mathrm{AB})$ and (CD). Even in moderate data sets, the number of potential T-patterns is very high. When, for instance, the potential number of event types is 100 , the number of potential patterns involving up to 10 event types is many orders of magnitude greater than $100^{10}$, if all possible time windows are also considered. Even for supercomputers, it becomes an impossible task to search for each possible temporal pattern separately.

To deal with this issue, simple patterns are detected first - that is, the identification of relationships between two event types such as the $(\mathrm{AB})$ or $(\mathrm{CD})$ relationship in Figure 2-whereas more complex patterns are detected as patterns of patterns (a so-called bottom-up search strategy). The simpler patterns (AB) and (CD) are detected first and, then the larger pattern $((A B)(C D))$ is detected as a combination of these. The new, larger pattern may then become part of even more complex patterns as it combines with other simple or complex patterns.

\section{Completeness Competition}

A further phase of T-pattern detection involves completeness competition between all the detected patterns. In this phase, the patterns that are less complete versions of one or more alternative patterns are deleted. For instance, during the detection process, a pattern $\mathrm{P}_{\mathrm{X}}=(\mathrm{ABCDE})$ may be partially detected as, for instance, (ACDE) or (BDE) or (ABCE). Since components of $\mathrm{P}_{\mathrm{X}}$ are miss-
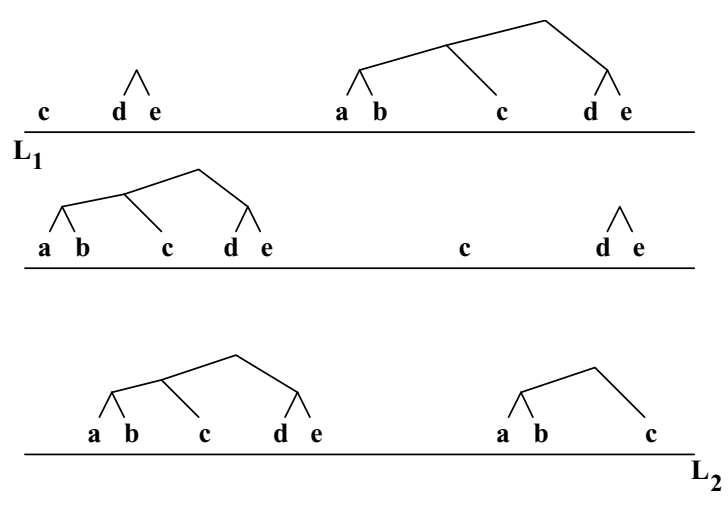

Figure 2. Repeated hierarchical real-time patterns. ing, these three patterns constitute less complete descriptions of the underlying patterning. Consequently, they are eliminated. This completeness competition ensures that only the most complete patterns survive and constitute the outcome of the analysis process (Borrie, Jonsson, \& Magnusson, 2002).

The T-pattern detection carried out by Theme in this research led to different types of data output, as will be described below.

\section{Theme Output}

Using the Theme software, we obtained three kinds of output data for our study.

The first output was the number of patterns detected for each observational period. That is, in each observed interaction, $N$ different patterns were detected, yielding an index of interaction synchrony: A higher number of patterns implied a more regular and ordered sequence of events.

The second kind of data output was pattern length, corresponding to the number of event types present in each pattern. For instance, the length of the pattern shown in the lower line in Figure 1 is four. Observation periods are classified by average pattern length, usually referred to as an index of interaction complexity.

The third and final output was the items in the raw data index (IR). This refers to the proportion of occurrences (\%) of a given event type recorded in the observation period. For instance, the proportion of occurrences of w, k, $\mathrm{a}, \mathrm{b}, \mathrm{c}$, and $\mathrm{d}$ on the upper line in Figure 1 give rise to an IR value for each of them.

\section{Objectives}

In the present research, observation was the method chosen to measure how hand gestures during conversation may be affected by culture in Icelandic and Italian people. Gestures were assumed to be the most socialized nonverbal modalities, since they are closely linked to language production in interactions (Bavelas, Chovil, Coates, $\&$ Roe, 1995). Primarily, a quantitative analysis was carried out on the gestures, considered as external variables, in order to measure how culture affects the typology and frequency of gestures (Duncan \& Fiske, 1977).

In the next step of analysis, we chose a structural approach with which to investigate the hidden temporal organization of behaviors and to explore how Icelandic and Italian subjects would sequentially organize their gesture production in conversation (Magnusson, 2000a).

A further aim of the research was to make a fresh contribution to observational studies on the relationship between culture and gestures by making use of an advanced tool, such as the Theme software.

\section{Setting and Procedure}

To ensure optimum control over contextual variables, the study was carried out in a laboratory setting, at the Communication Psychology Lab of the Catholic University of Milan and at the Human Behavior Laboratory of Reykjavík University. The subjects took part in a coopera- 
tive task: Each pair was asked to think up and talk about an advertisement for a bubble bath as if they were creative advertising experts.

Their conversations had a fixed duration of $15 \mathrm{~min}$ and were audio and video recorded.

Each video sequence was then coded frame by frame, using the Theme Coder software (Magnusson, 1993, 2000a). The coding was done separately for each behavior unit, and both the beginning frame and the ending frame of each behavior unit were marked.

The grid used for observation of the interactions is shown in Table 1. This grid is made up of 48 behavior units, divided into nine categories and describing different types of body movements involving hands, arms, head, fingers, trunk, shoulders, direction of gaze, and facial expressions; turn-taking was also included as a behavioral unit. The majority of the behavior units were taken from grids used in previous research on nonverbal behavior (Argyle, 1975; Ekman \& Friesen, 1978; Grammer et al., 1998; Mehrabian, 1972; Morris, 1977). The coded behavior was then analyzed with Theme, in order to detect T-patterns.

Only a segment of each interaction was taken into consideration for behavior coding-namely, the 3 central minutes. The duration of each interaction was divided in half, and the $90 \mathrm{sec}$ before and after the halfway point were considered for coding. This limitation was due to the time-consuming nature of behavior coding.

\section{Results}

Two different analyses were carried out on the data collected, following two methodological approaches: quantitative (external variables) and structural.

The quantitative approach. A first multivariate ANOVA was performed on the IR data for each behavior unit coded. A significant main effect was found for the culture variable $[F(1,29)=14.01, p<.0001]$; culture seems to influence the production of gestures, in terms of both frequency and typology. The Italian subjects displayed a higher gesture frequency than did the Icelanders, as is shown in Figure 3.

Table 1

Nonverbal Behavior Grid Used in Study

\begin{tabular}{ll} 
& Nonverbal Behavior Grid Used in Study \\
\hline Category & \multicolumn{1}{c}{ Behavior Units Description } \\
\hline Hands & $\begin{array}{l}\text { rotation, hand contact, crossed hands, hand on face, hand } \\
\text { on body, hand on chair, hand on hair, palm inward, palm } \\
\text { forward, palm down, palm upward, palm back, palm } \\
\text { outside, fist, bag-hand, mirror-hands, rubbing hands, } \\
\text { ax-hand, hitchhiking, ring, hands away, ball-hands } \\
\text { pointing, counting, negation, pianist } \\
\text { arms upward, crossed arms, arm forward, open arms, } \\
\text { dangling arms, arms back, bend arms } \\
\text { Fingers }\end{array}$ \\
Arms & $\begin{array}{l}\text { head forward, head down, head back, tilt head left/right, } \\
\text { tilt head up/down, negation by head } \\
\text { trunk forward, tilt trunk left/right, trunk back, swinging }\end{array}$ \\
Trunk & smile, laugh \\
Face & avert gaze \\
Gaze & shrug shoulders \\
Shoulders & speak \\
Turn &
\end{tabular}

In particular, movements of the palm of hand (palm inward, palm upward, palm back, and mirror-hands) and self-contact gestures (hand on hair, hand on face, and palm contact) were observed mostly in Italian interactions, whereas a higher number of crossed-hand gestures were coded in Icelandic interactions.

Moreover, distinctive gestures emerged, attributable to cultural differences. For instance, the Italian subjects displayed specific gestures, such as bag-hand and ring, that were never used by the Icelanders. On the other hand, the Icelandic subjects used ball-hands and pianist gestures that were never present in the Italians.

The structural approach. The second step consisted of a structural analysis of the T-patterns detected. For each observed interaction, a high number of patterns was computed by Theme: Specifically, an average of 300 T-patterns was identified, each recurring at least three times in the analyzed observational period. ${ }^{1}$

Each detected T-pattern included at least two event types. The event types included in the patterns referred not only to gestures, but also to other behavior units (movements of arms, head, fingers, trunk, shoulders, direction of gaze, and facial expressions) occurring along with gestures.

By way of illustration, three different T-patterns including three typical gestures that appear to be cultural discriminators are described in Figures 4-6.

The picture and graph in Figure 4 refer to the bag-hand gesture displayed by an Italian dyad of friends. The graph details the T-pattern, which recurred three times during the observation period. This T-pattern is made up of five event types, annotated using the standard abbreviations. Specifically, in this behavioral sequence, the first subject (on the right) begins a speaking turn (subjectA, b, Tu); while he is speaking, the second subject (on the left) also begins a speaking turn (subjectB, b, Tu); while they are both speaking, the second subject displays the bag-hand gesture (subjectB, b, M15). Finally, the first subject ends the speaking turn (subjectA, e, Tu), and the second subject ends the trunk forward movement (subjectB, e, Bu1). The picture shows a screenshot of a video frame of the recorded interaction corresponding to this behavioral sequence.

Figure 5 shows a picture and a graph of a pattern including a typical gesture detected in Icelandic people, the pianist. This T-pattern is a good deal more complex than the previous one, since it is composed of 12 event types. The behavioral sequence involves both interactants. In particular, it includes the beginning and ending of the speaking turn, inclination of the head, and displaying of the pianist gesture by one interactant, while the other subject looks away.

Finally, Figure 6 shows the second Icelandic gesture detected in this study, the ball-hands gesture. This T-pattern is made up of eight events that include the ball-hands gesture displayed by an interactant and a contemporary lookdance, as the 2 subjects repeatedly make eye contact and then look away.

\section{Discussion}

The study described in this article confirms the importance of considering different theoretical and methodolog- 


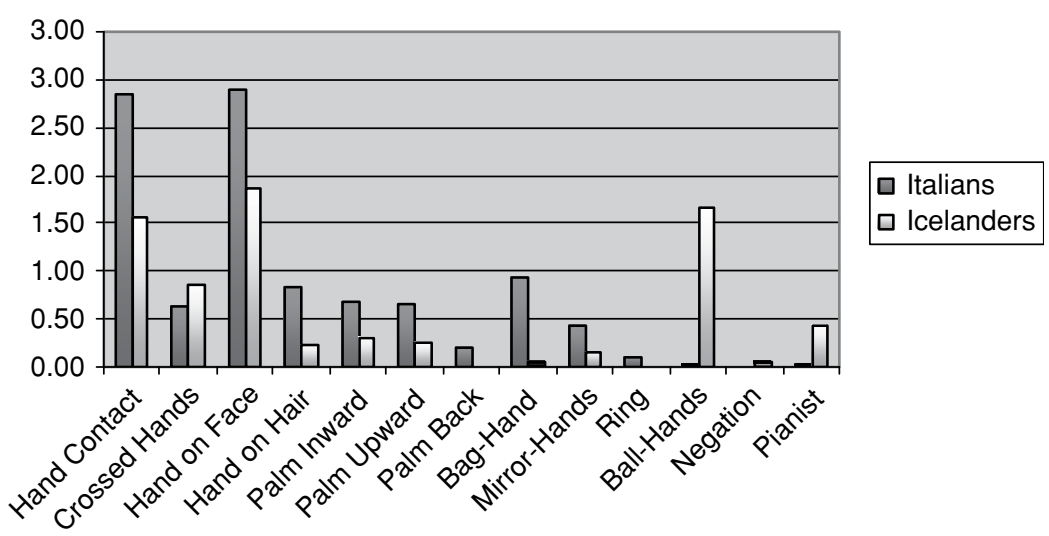

Figure 3. Means of the gesture frequencies displayed by the Italians and the Icelanders.

ical issues when measuring behavior, given its unquestionable degree of complexity. Indeed, in this research, a quantitative approach to behavior was integrated with a structural analysis. The former highlighted the unhidden (external) differences between the Icelandic and the Italian subjects' gestures. These external variables were found to be culturally influenced, in line with the social pragmatic perspective (Goodwin, 2000). The latter analysis revealed the hidden organization of behavior. Both Icelandic and Italian interactions appear to make up organized and complex systems, where a huge number of events are constantly happening and recurring. A solely quantitative analysis was insufficient for understanding these systems; patterning and the hidden structure of behavior also needed to be considered. In this regard, pattern detection with Theme represents a substantial methodological progression from solely quantitative to structural data analysis. It should be pointed out that most of the behavioral sciences have traditionally included repeated patterns among their central concerns. However, tools based on relevant and adequate models and methods have been difficult to find in this area.

By considering not only order, but also real time, when searching for hidden interaction structure, Theme demon-

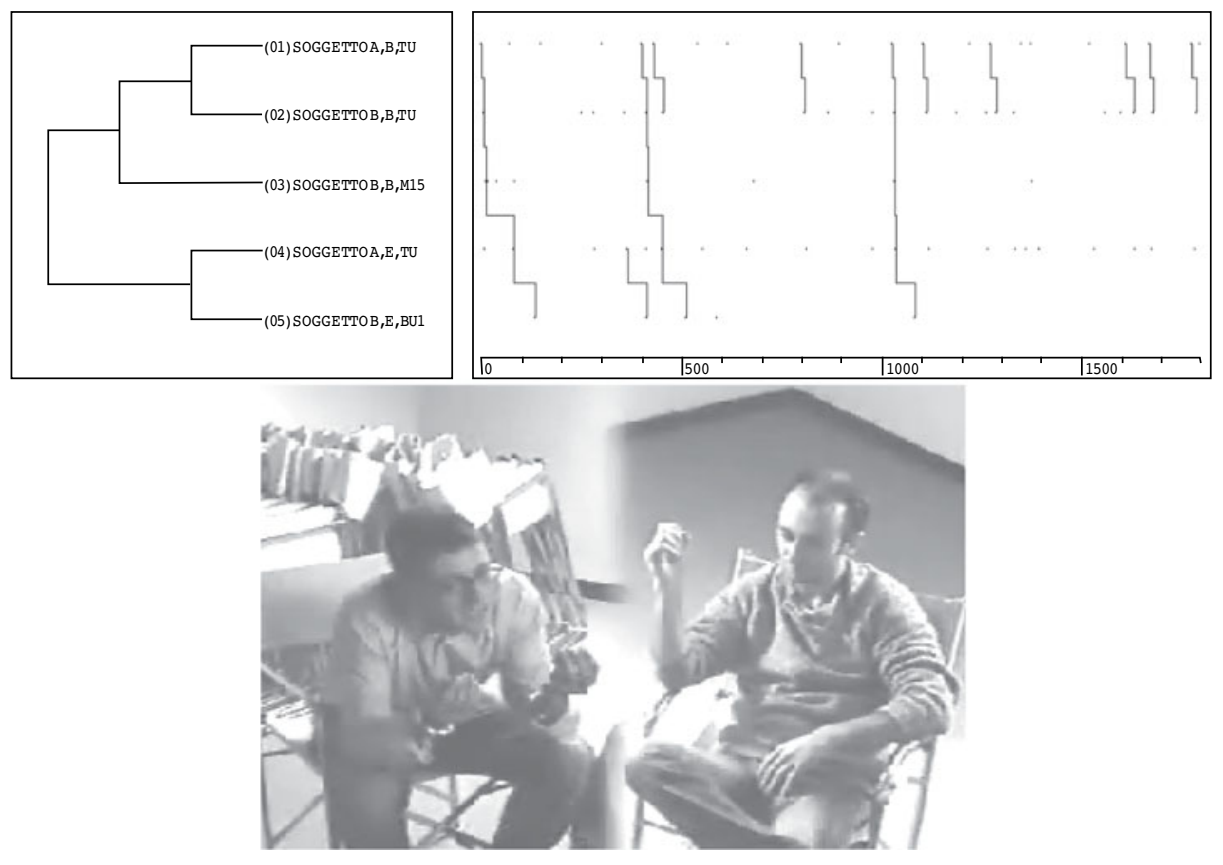

Figure 4. A T-pattern detected in an Italian dyad that includes the bag-hand gesture and an interaction frame extracted from this behavioral sequence. The T-pattern is formed by five events: (1) subjectA, b, turn; (2) subjectB, b, turn; (3) subjectB, b, bag-hand; (4) subjectA, e, turn; (5) subjectB, $b$, trunk forward. 


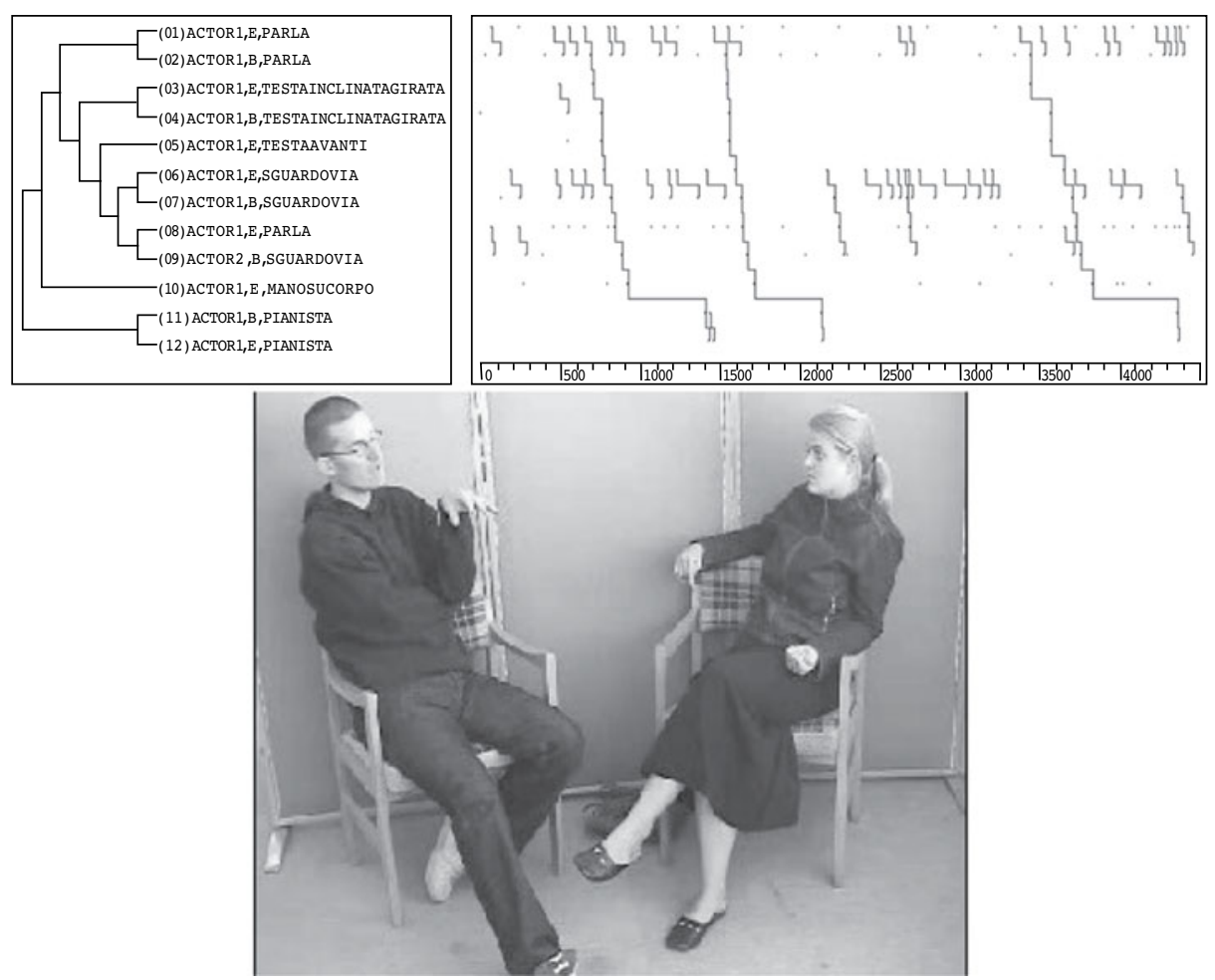

Figure 5.A T-pattern detected in an Icelandic dyad that includes the pianist gesture and an interaction frame extracted from this behavioral sequence. The T-pattern is formed by 12 events: (1) subjectB, e, turn; (2) subjectA, b, turn; (3) subjectA, b, tilt head; (4) subjectA, e, tilt head; (5) subjectA, b, head forward; (6) subjectA, e, look away; (7) subjectB, b, look away; (8) subjectA, e, turn; (9) subjectB, b, look away; (10) subjectA, e, hand on body; (11) subjectA, b, pianist; (12) subjectA, e, pianist.

strates how regularities in such interactions may outcrop by the use of adequate methods. The patterns thus discovered can contribute significantly to behavior measurement, by providing in-depth information on the temporal features and rhythmic organization of behavior (Magnusson, 2005).

In our study, behavior regularity emerged as a stable feature of human communicative exchanges. A high number of behavioral patterns were found in all the observed interactions. This is consistent with the assumption that even though communication may appear chaotic and confused, especially given its spontaneous nature, in reality it is highly regulated and organized, on the basis of shared cultural standards (Anolli, 2004).

The results of the quantitative analysis lent support to the cultural hypothesis, according to which Icelandic and Italian conversation would be characterized by distinctive typologies of gestures. In general, the Italian subjects regularly called on more varied and more dynamic gestures than did the Icelanders (for instance, movement of the palms up, back, inward, and so on). Conversely, the Icelandic subjects typically made use of close and spatially limited gestures, such as crossed hands, pianist, and ball-hands.

Furthermore, the Italian subjects produced self-contact gestures more frequently than did their Icelandic counter- parts. These gestures may be attributed to the generally restless and excited style of Italian people in conversation.

Finally, some gestures, such as bag-hand and ring, were displayed only by the Italian subjects, whereas the pianist and the ball-hand gestures were shown only by the Icelanders.

Summing up, the data appear to support the cultural perspective in social pragmatics, whereby the tangled network between language and body movements is produced in conformity to cultural standards.

On the whole, the methodological approach of this study proved to be functional for nonverbal behavior detection and measurement in conversational settings. Furthermore, it seems to provide an analysis procedure capable of taking into account cultural variables, thus representing an attempt to face the challenges arising from behavioral complexity.

Nonetheless, some limitations of the study must be acknowledged. The primary drawback was the artificiality of the laboratory setting and the consequent decrease in ecological validity. Furthermore, only nonverbal behavior was considered; no connections between specific gestures and correspondent linguistic topics were examined. A multimodal interaction analysis using a similar methodological approach can be recommended as an interesting topic for future studies. 


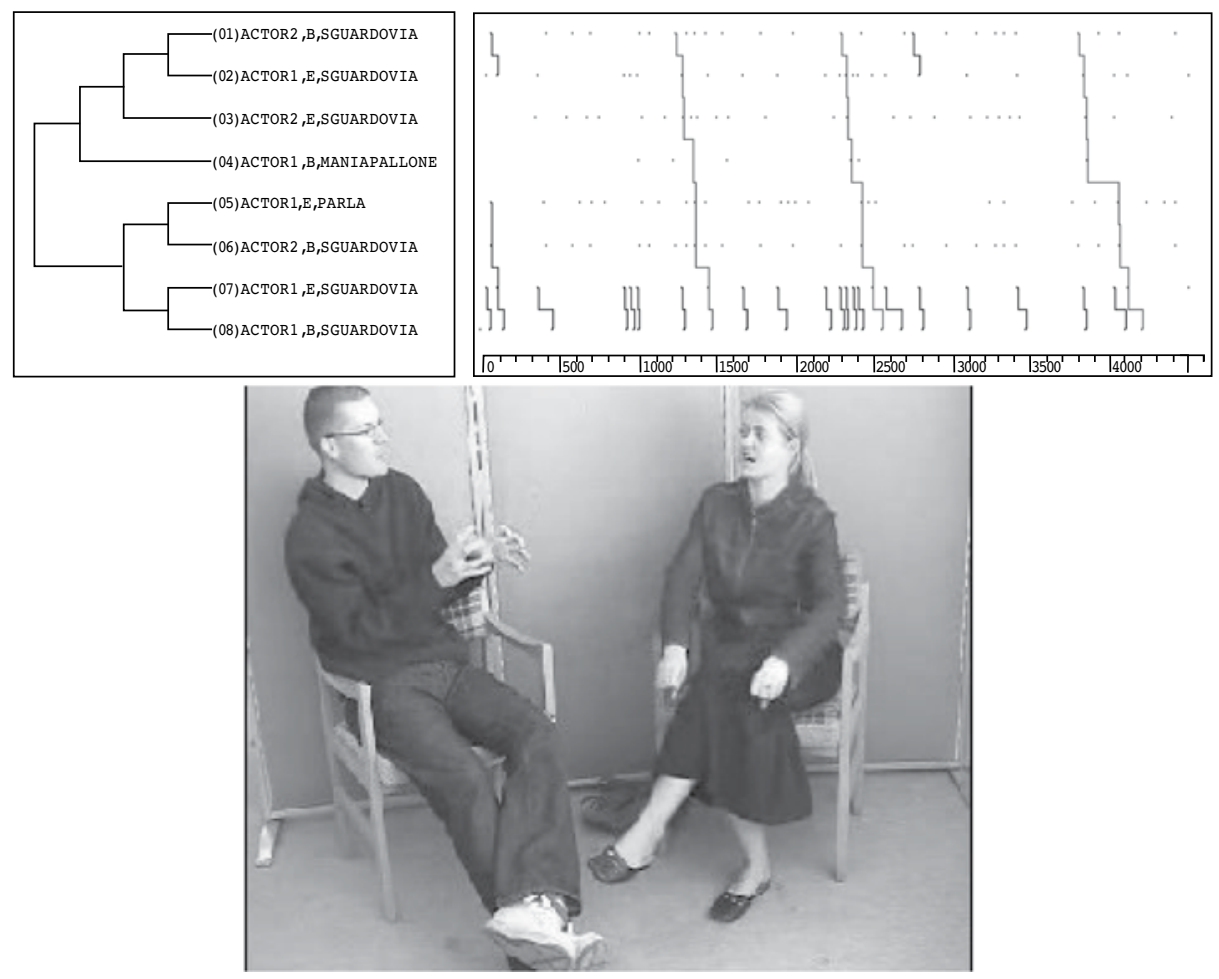

Figure 6. A T-pattern detected in an Icelandic dyad that shows the ball-hands gesture and an interaction frame extracted from this behavioral sequence. The T-pattern is formed by eight events: (1) subjectB, b, look away; (2) subjectA, e, look away; (3) subjectB, e, look away, (4) subjectA, b, ball-hands; (5) subjectA, e, turn; (6) subjectB, b, look away; (7) subjectA, e, look away; (8) subjectA, b, look away.

\section{REFERENCES}

ANGUERA, M. T. (2003). Observational methods (general). In R. FernándezBallesteros (Ed.), Encyclopedia of psychological assessment (Vol. 2, pp. 632-637). London: Sage.

Anolli, L. (2004). Psicologia della cultura. Bologna: Il Mulino.

Anolli, L., Riva, G., Duncan, S., JR., \& Magnusson, M. S. (EDs.) (2005). The hidden structure of interaction: From neurons to culture patterns. Amsterdam: IOS Press.

Argyle, M. (1975). Bodily communication. London: Methuen.

Bavelas, J., Chovil, N., Coates, L., \& Roe, L. (1995). Gestures specialized for dialogue. Personality \& Social Psychology Bulletin, 21, 394-405.

BERNIERI, F. J., \& Rosenthal, R. (1991). Interpersonal coordination: Behavior matching and interactional synchrony. In R. S. Feldman \& B. Rimè (Eds.), Fundamentals of nonverbal behavior (pp. 401-432). Cambridge: Cambridge University Press.

BIRDWHISTELL, R. L. (1970). Kinesics and context: Essays on body motion communication. Philadelphia: University of Pennsylvania Press.

Bloomfield, J., Jonsson, G. K., Polman, R., Houlahan, K., \& O'Donoghue, P. (2005). Temporal pattern analysis and its applicability in soccer. In L. Anolli, G. Riva, S. Duncan, Jr., \& M. S. Magnusson (Eds.), The hidden structure of interaction: From neurons to culture patterns (pp. 237-251). Amsterdam: IOS Press.

Borrie, A., Jonsson, G. K., \& Magnusson, M. S. (2002). Temporal pattern analysis and its applicability in sport: An explanation and exemplar data. Journal of Sports Sciences, 20, 845-852.

Duncan, S., JR., \& Fiske, D. W. (1977). Face-to-face interaction: Research, methods, and theory. Hillsdale, NJ: Erlbaum.

EIBL-EIBESFELDT, I. (1970). Ethology: The biology of behavior (E. Klinghammer, Trans.). New York: Holt, Rinehart \& Winston.
EkMAN, P., \& Friesen, W. V. (1978). Facial action coding system: Investigator's guide. Palo Alto, CA: Consulting Psychologists Press.

Giles, H., Coupland, N., \& Coupland, J. (1991). Accommodation theory: Communication, context and consequences. In H. Giles, J. Coupland, \& N. Coupland (Eds.), Context of accommodation (pp. 1-68). Cambridge: Cambridge University Press.

GiLes, H., \& Sмiтh, P. M. (1979). Accommodation theory: Optimal levels of convergence. In H. Giles \& R. St. Clair (Eds.), Language and social psychology (pp. 45-65). Oxford: Blackwell.

GoodwIN, C. (1981). Conversational organization: Interaction between speakers and hearers. New York: Academic Press.

Goodwin, C. (2000). Action and embodiment within situated human interaction. Journal of Pragmatics, 32, 1489-1522.

Goodwin, C. (2002). Time in action. Applied Linguistics, 43, 1-53.

Grader, M. (2003). Expressive timing and interaction synchrony between mothers and infants: Cultural similarities, cultural differences, and the immigration experience. Cognitive Development, 18, 533554.

Graham, J. A., \& Argyle, M. (1975). A cross-cultural study of the communication of extra-verbal meaning by gestures. International Journal of Psychology, 10, 56-67.

Grammer, K., Kruck, K. B., \& Magnusson, M. S. (1998). The courtship dance: Patterns of nonverbal synchronization in opposite-sex encounters. Journal of Nonverbal Behavior, 22, 3-29.

HaLl, E. T., \& HALL, M. R. (1999). Monochronic and polychronic time. In L. K. Guerrero, J. A. DeVito, \& M. L. Hecht (Eds.), The nonverbal communication reader: Classic and contemporary readings (2nd ed., pp. 237-240). Prospect Heights, IL: Waveland.

HigGins, E. T. (2000). Does personality provide unique explanations for behavior? Personality as cross-person variability in general principles. European Journal of Personality, 14, 391-406. 
MaGnusson, M. S. (1993). Hidden real-time patterns in intra- and interindividual behavior: Description and detection. European Journal of Psychological Assessment, 12, 112-123.

Magnusson, M. S. (2000a). Discovering hidden time patterns in behavior: T-patterns and their detection. Behavior Research Methods, Instruments, \& Computers, 32, 93-110.

Magnusson, M. S. (2000b). Modeling complex real-time behavioral streams as optimised sub-sets of mutually exclusive and nested Tpatterns. In Proceedings of Measuring Behavior 2000, 3rd International Conference on Methods and Techniques in Behavioral Research (pp. 168-171). Nijmegen: Blackwell.

MaGnusson, M. S. (2005). Understanding social interaction: Discovering hidden structure with model and algorithms. In L. Anolli, G. Riva, S. Duncan, Jr., \& M. S. Magnusson (Eds.), The hidden structure of interaction: From neurons to culture patterns (pp. 3-22). Amsterdam: IOS Press.

McGrew, W. C. (1972). An ethological study of children's behavior. New York: Academic Press.

Mehrabian, A. (1972). Nonverbal communication. Chicago: AldineAtherton.

Merten, J., \& Schwab, F. (2005). Facial expression patterns in common and psychotherapeutic situations. In L. Anolli, G. Riva, S. Duncan, Jr., $\&$ M. S. Magnusson (Eds.), The hidden structure of interaction: From neurons to culture patterns (pp. 149-158). Amsterdam: IOS Press.

MorRIs, D. (1977). Manwatching: A field guide to human behaviour. London: Cape.

OcHs, E. (1992). Indexing gender. In A. Duranti \& C. Goodwin (Eds.), Rethinking context: Language as an interactive phenomenon (pp. 336358). Cambridge: Cambridge University Press.

Zimmerman, D., \& West, C. (1975). Sex roles, interruptions and silences in conversation. In B. Thorne \& N. Henley (Eds.), Language and sex: Difference and dominance (pp. 105-129). Rowley, MA: Newbury House.

\section{NOTE}

1. The pattern detection parameters were $p=.005$ for significance level and minimum occurrences $=3$.

(Manuscript received September 28, 2005; revision accepted for publication January 28,2006 .) 